\title{
Disfunción muscular respiratoria en pacientes llevados a cirugía cardiovascular
}

\author{
Diana Cáceres ${ }^{a, b, c, d, *}$, Karenth Meneses ${ }^{e}$, Alba Ramírez-Sarmiento ${ }^{a, b, d}$, \\ Clara Domínguez $^{a, b, d}$, Anderson Bermon ${ }^{b}$, Antonio Figueredo ${ }^{f}$, Rubén Duarte ${ }^{a}$ \\ y Mauricio Orozco-Levi ${ }^{\text {a,b,c,d,g,h }}$
}

\author{
a Servicio de Neumología, Fundación Cardiovascular de Colombia, Floridablanca, Santander, Colombia \\ b Unidad de Investigaciones, Fundación Cardiovascular de Colombia, Floridablanca, Santander, Colombia \\ c CEXS-Universidad Pompeu Fabra, Barcelona, España \\ ¿ Grupo de Investigación EMICON, Cod. COLCIENCIAS COL01372334, Bucaramanga, Colombia \\ e Universidad Autónoma de Bucaramanga, Bucaramanga, Colombia \\ f Servicio de Cirugía Cardiovascular, Fundación Cardiovascular de Colombia, Floridablanca, Santander, Colombia \\ g Facultad de Medicina, Universidad de Santander, Bucaramanga, Colombia \\ h CIBER de Enfermedades Respiratorias, ISCIII, España
}

Recibido el 26 de octubre de 2015; aceptado el 15 de mayo de 2016

Disponible en Internet el 1 de julio de 2016

\section{PALABRAS CLAVE \\ Cirugía cardiaca; Complicaciones; \\ Pulmón}

\begin{abstract}
Resumen
Objetivo: Evaluar la presencia de las alteraciones en la fuerza muscular respiratoria en pacientes programados para la cirugía cardiovascular, el impacto de la cirugía sobre la función muscular respiratoria y su relación con las alteraciones ventilatorias en el postoperatorio mediato. Métodos: Estudio observacional prospectivo. Se realizaron pruebas de funcionalismo respiratorio (espirometría) y pruebas de fuerza muscular respiratoria (inspiratoria: presión inspiratoria máxima y espiratoria: presión espiratoria máxima) en dos momentos: preoperatorio inmediato ( $2 \pm 1$ días precirugía) y postoperatorio mediato $(2 \pm 1$ días, antes del alta). Las complicaciones respiratorias incluyeron: la ventilación mecánica prolongada, el tromboembolismo pulmonar, el neumotórax, el hemotórax, la neumonía, el derrame pleural, el edema pulmonar y las atelectasias.

Resultados: Se evaluaron 30 pacientes adultos $(n=30)(62 \pm 12$ años). En el preoperatorio la capacidad espirométrica disminuyó en un 54\% (35\% alteración obstructiva, 19\% no-obstructiva) y la debilidad inspiratoria y espiratoria fue confirmada en un 67 y $100 \%$, respectivamente. Las complicaciones respiratorias estuvieron presentes en un $93 \%$ (con mayor frecuencia la atelectasia y el derrame pleural). El deterioro postoperatorio grave de la presión inspiratoria máxima incrementó 10 veces el riesgo de atelectasia $(O R=10, I C 95 \% 0,85-117,02 ; p=0,067)$. Al alta, la
\end{abstract}

\footnotetext{
* Autor para correspondencia.

Correo electrónico: dianacaceres@fcv.org (D. Cáceres).
} 
fracción de eyección del ventrículo izquierdo fue $29 \%$ menor, la capacidad vital forzada $-32 \%$, la presión inspiratoria máxima $-32 \%$ y la presión espiratoria máxima $-32 \%$ con respecto al valor inicial.

Conclusiones: Los pacientes llevados a la cirugía cardiovascular tienen una insospechada disfunción de los músculos respiratorios que empeora con la cirugía y la hospitalización, que se asocia a un incremento del riesgo de complicaciones respiratorias.

(c) 2016 Sociedad Colombiana de Cardiología y Cirugía Cardiovascular. Publicado por Elsevier España, S.L.U. Este es un artículo Open Access bajo la licencia CC BY-NC-ND (http:// creativecommons.org/licenses/by-nc-nd/4.0/).

\section{KEYWORDS}

Cardiac surgery; Complications;

Lung

\section{Respiratory muscle dysfunction in patients undergoing cardiovascular surgery}

\begin{abstract}
Motivation: To assess the presence of alterations in respiratory muscle strength in patients with a programmed cardiovascular surgery, the impact of the surgery on respiratory muscle function and their relationship with ventilatory alterations during mediate postoperative period.

Methods: Prospective observational study. Respiratory function tests (spirometry) and respiratory muscle strength tests (inspiratory: maximum inspiratory pressure, and expiratory: maximum expiratory pressure) were conducted in two moments: immediate preoperative ( $2 \pm 1$ days before surgery) and mediate postoperative period ( $2 \pm 1$, before being discharged). Respiratory complications included prolonged mechanical ventilation, pulmonary thromboembolism, pneumothorax, hemothorax; pneumonia; pleural effusion; pulmonary edema and atelectases. Results: 30 adult patients were assessed $(n=30)$ (ages $62 \pm 12$ ). Spirometry performance decreased by $54 \%$ in the preoperative period ( $35 \%$ obstructive alteration, $19 \%$ non-obstructive) and inspiratory and expiratory weakness was confirmed at 67 and $100 \%$ respectively. Respiratory complications were present in $93 \%$ (more frequently atelectasis and pleural effusion). Severe postoperative deterioration of maximum inspiratory pressure increased by 10 -fold the risk of atelectasis $(O R=10$, IC $95 \% 0.85-117.02 ; p=0.067)$. Upon discharge, ejection fraction of the left ventricle was $29 \%$ lower, forced vital capacity $-32 \%$, maximum inspiratory pressure $-32 \%$ and maximum expiratory pressure $-32 \%$ with regards to baseline values.

Conclusion: Patients undergoing cardiovascular surgery suffer an unsuspected respiratory muscle dysfunction that worsens with surgery and hospitalisation, associated to a increase of the risk of respiratory complications.

(c) 2016 Sociedad Colombiana de Cardiología y Cirugía Cardiovascular. Published by Elsevier España, S.L.U. This is an open access article under the CC BY-NC-ND license (http:// creativecommons.org/licenses/by-nc-nd/4.0/).
\end{abstract}

\section{Introducción}

Durante los últimos años se ha demostrado la presencia de la disfunción muscular respiratoria en pacientes con compromiso cardiovascular y su relación con la aparición de las complicaciones pulmonares postoperatorias en cirugía cardiaca, tales como las atelectasias y la neumonía ${ }^{1-5}$, las cuales representan las causas más comunes de la mortalidad y la morbilidad en el postoperatorio en este tipo de cirugía, ocasionando la prolongación de la estancia hospitalaria y el aumento en los recursos y costos utilizados ${ }^{1,2,6}$.

La disfunción muscular respiratoria en pacientes con compromiso cardiaco ha sido relacionada con las alteraciones estructurales y metabólicas, particularmente, en los músculos inspiratorios, ocasionando pérdida de la fuerza y resistencia muscular ${ }^{3,7-11}$, lo cual está directamente relacionado con el empeoramiento de la disnea, síntoma característico en dicha población ${ }^{2}$. El estado preoperatorio de los músculos respiratorios en pacientes con compromiso cardiaco ha sido estudiado en el contexto de la cirugía cardiovascular, siendo la cirugía de revascularización miocárdica y de cambio valvular las más estudiadas, en donde se evidencia la presencia de la disfunción muscular respiratoria y su relación con la aparición de complicaciones pulmonares postoperatorias ${ }^{1,6,7,9}$, y a su vez se describe el papel de la presión inspiratoria máxima (PIM) como factor protector en la reducción de la estancia hospitalaria durante el postoperatorio de la cirugía torácica ${ }^{12}$.

Diversos factores propios de la cirugía cardiaca y las intervenciones realizadas en el cuidado del paciente durante el postoperatorio, predisponen a cambios de la función pulmonar. La ventilación mecánica representa una de las situaciones más comunes en los pacientes durante el postoperatorio de la cirugía cardiaca, en donde el deterioro de la fuerza y resistencia del diafragma y los músculos accesorios de la inspiración, generan un mecanismo fisiopatológico que se asocia a la hipoventilación alveolar y por consiguiente a un destete prolongado de la misma ${ }^{13}$. El efecto de la atrofia que genera la ventilación mecánica a los músculos importantes de la respiración, favorece la 
disfunción de la musculatura respiratoria y la prolongación de la liberación de la ventilación mecánica, agravando el cuadro respiratorio. En el caso de los músculos espiratorios, esta disfunción precipita que la tos sea ineficaz y su participación se deteriora en los movimientos respiratorios activos $^{14}$. Igualmente, factores como el dolor postoperatorio juegan un papel importante en la alteración de la función respiratoria, generando un patrón respiratorio superficial, lo que conlleva a una disminución de los volúmenes pulmonares, siendo los más afectados la capacidad vital forzada y el volumen espiratorio máximo en el primer segundo ${ }^{15}$. Asimismo, induce una inhibición voluntaria de la tos, que junto con la ventilación mecánica conlleva a un colapso alveolar, favoreciendo la retención de las secreciones y por consiguiente la aparición de infecciones como la neumonía ${ }^{16}$.

La hipótesis de este estudio plantea que la presencia de la disfunción muscular respiratoria en pacientes sometidos a la cirugía cardiovascular electiva por patología cardiaca, conlleva al desarrollo de las complicaciones pulmonares en el postoperatorio mediato. Teniendo en cuenta lo mencionado anteriormente, el objetivo de este estudio está orientado a evaluar tres aspectos importantes: a) La presencia de alteraciones en la fuerza muscular respiratoria y periférica en pacientes programados para toracotomía electiva por patología cardiovascular, b) El impacto de la cirugía sobre la función muscular respiratoria, y c) Su relación con las alteraciones ventilatorias en el postoperatorio mediato.

\section{Materiales y métodos}

Se realizó un estudio observacional prospectivo durante los meses de septiembre a diciembre de 2014, tiempo durante el cual se contó con una terapeuta respiratoria para la realización de las pruebas e incluyó un número total de 146 pacientes programados para cirugía cardiovascular, de los cuales se valoraron 30 pacientes que cumplían con los criterios de inclusión y estaban acorde al cronograma del estudio. Todos los pacientes eran mayores de 18 años de edad y se encontraban en protocolo prequirúrgico de cirugía cardiovascular hospitalizados o ambulatorios que accedieron a participar voluntariamente en el estudio. Los datos fueron obtenidos del registro de pacientes programados para la cirugía cardiovascular durante el segundo semestre de 2014 en la Fundación Cardiovascular de Colombia.

Se excluyeron los pacientes que no pudieron realizar la maniobra espirométrica, que al momento de su valoración estuvieran en: un procedimiento, pacientes con foco infeccioso, hemodinámicamente inestables, con alteraciones cognitivas, con patologías respiratorias severas o con diagnóstico de delirium. En todos los casos se obtuvo el consentimiento informado por escrito. Se informó a los pacientes y familiares sobre las características del estudio, el cual se llevó a cabo siguiendo rigurosamente las recomendaciones éticas internacionales para investigación médica en humanos de acuerdo con las normas recogidas en la declaración de Helsinki y la resolución 008430 de 1993.

Se realizó a cada paciente las siguientes mediciones:

- Espirometría simple: Se utilizó el espirómetro portátil Microlab $^{\circledR}$.
- Fuerza muscular respiratoria: presión inspiratoria máxima (PImáx.) y presión espiratoria máxima (PEmáx.), para lo cual se utilizó el equipo portátil Microlab ${ }^{\circledR}$.

Para obtener información válida se tuvieron en cuenta las recomendaciones de la normativa SEPAR (Sociedad Española de Neumología y Cirugía de Tórax) para la realización de las pruebas pulmonares ${ }^{17}$.

Adicionalmente, se registraron las variables demográficas, exposicionales y quirúrgicas. Estas pruebas se realizaron en dos momentos: 1. Preoperatorio inmediato ( $2 \pm 1$ días antes de la cirugía) y 2 . Postoperatorio mediato ( $2 \pm 1$ días, antes del alta) y fueron llevadas a cabo en las diferentes unidades donde se encontraban los pacientes hospitalizados.

Las complicaciones respiratorias fueron definidas según el protocolo de manejo del paciente sometido a cirugía cardiovascular de la Fundación Cardiovascular de Colombia el cual se basa en las definiciones de "The Society of Thoracy Surgeons" (STS), dentro de las cuales se incluyen: la ventilación mecánica prolongada (> 24 horas), el tromboembolismo pulmonar (TEP), el neumotórax, el hemotórax, la neumonía y el derrame pleural ${ }^{18}$. Para efectos del presente estudio, se añadieron, además, el edema pulmonar y las atelectasias considerados por el presente grupo de relevancia clínica y apoyados en la STS, quienes recomiendan incluir las complicaciones que se consideren de gran impacto por diferentes motivos en una institución de salud. Esta información se resume en el Anexo 1.

Criterios de debilidad respiratoria: para identificar la debilidad respiratoria se utilizaron las ecuaciones propuestas por Morales y Cols., las cuales son las más utilizadas en trabajos previos del grupo en estudio y por muchos otros en poblaciones latinas. Esto aporta mayor posibilidad de generalizar y facilitar comparaciones con nuestros resultados. Teniendo en cuenta los porcentajes de los valores medidos con relación a las ecuaciones de referencia, se consideraron como normales las medidas mayores al $80 \%$ del valor predicho según lo descrito por Hautmann y Cols., en el año 2000 y Evans y Cols., en el año 2009, quienes refieren este porcentaje como límite para definir la debilidad respiratoria independientemente de los valores de referencia utilizados $^{19,20}$.

\section{Análisis estadístico}

Primero se realizó un análisis descriptivo del total de las variables, los datos se reportaron con porcentajes y frecuencias para variables categóricas y medidas de tendencia central y desviación para las cuantitativas. Luego se llevó a cabo un análisis bivariado con pruebas de chi2, test exacto de Fisher, $T$ de student y $U$ de Mann Whitney según correspondiera tomando como variable dependiente los cambios presentados entre el momento prequirúrgico y el posquirúrgico tanto para la PIM como para la PEM. Por último se realizó un análisis longitudinal multinivel con regresión logística para analizar las diferencias. Se utilizó como herramienta de captura el software de Excel exportando los datos a Stata versión 12 para los análisis. El poder del estudio para el análisis de las diferencias de medidas repetidas fue del $95 \%$. 


\section{Resultados}

Se incluyeron 30 pacientes en total, 8 mujeres y 21 hombres con un promedio de edad de 62,7 años (media 59,5 $\pm 10,1$ ). La estancia en la unidad de cuidados intensivos fue en promedio de 4,7 (media $5 \pm 2,1$ ) y la estancia general total de 20 días (media 22,4 $\pm 6,5$ ). El promedio de horas, el tiempo de ventilación mecánica fue de 6 horas (media 12,8 $\pm 14,6$ ), donde solo un paciente tuvo ventilación mecánica prolongada (48 horas). El manejo del dolor postoperatorio se realizó en todos los casos mediante la bomba de analgesia controlada por el paciente (PCA) de fentanilo, según protocolo institucional y se realizaron las pruebas funcionales en ausencia de dolor. El $56 \%$ de los pacientes tenía antecedentes de tabaquismo (activo o pasado) con un índice tabáquico promedio de 3,8 . Un $20 \%$ presentaba exposición crónica al humo de biomasa en el domicilio. El $16 \%$ de los pacientes mostraba un peso anormal (BMI: $10 \%>30$, y $6 \%<20 \mathrm{~kg} / \mathrm{m}^{2}$ ). En la tabla 1 se muestran las demás variables descriptivas de la población.

La capacidad espirométrica prequirúrgica estuvo disminuida en el 54\% de los casos: $35 \%$ alteración obstructiva, $19 \%$ no-obstructiva; $\mathrm{FEV}_{1}$ entre $32-76 \%$ según los valores de referencia de Crapo. Con respecto a la presión inspiratoria máxima, los pacientes que fueron programados para la cirugía cardiaca presentaron alta prevalencia de debilidad muscular con una PImáx., promedio de 68,6\% de los valores de referencia de Morales y Cols. ${ }^{21}$ (DS: 21,4, (rango: 34-122), Rango Inter Cuartil (RIC) (58-83)), para un total de 20 casos con criterio de debilidad (69\% de la población) (fig. 1A). El escenario fue peor para la PEmáx., en la que se encuentra un promedio de 51\% (DS: 14,5; (rango: 15-77), RIC (43-59)), todos los pacientes se encontraban con un rango inferior al $80 \%$ de los valores de referencia (fig. 1B). Se realizó un análisis longitudinal de los datos encontrandos, la diferencia estadísticamente significativa entre los valores de referencia de Morales previos a la cirugía y posteriores a esta ( $p<0,05$, IC: $x-y)$ tanto en la PImáx. como en la PEmáx. Se realizó una variable dicotómica con la diferencia de las PIM para que fuera de más fácil comprensión teniendo como punto de referencia el presentar una diferencia superior a 19 entre la PIM postquirúrgica y la PIM prequirúrgica (este valor sale de la mediana de las diferencias de los dos valores). Este valor se tomó como significativo pese a la p superior a 0,067 y los valores del intervalo de confianza, debido al tamaño reducido de la muestra. La variable de cambio de la PImáx., se tomó cuantitativa analizada por medio de una regresión logística por lo que el efecto no se determina desde un punto basal, sino por la magnitud de la diferencia entre la PImáx. posquirúrgica y la PImáx. prequirúrgica.

Las complicaciones postoperatorias encontradas en los pacientes fueron: atelectasia: $67 \%$, neumotórax $24 \%$, derrame pleural $54 \%$, neumonía $7 \%$ y edema pulmonar $10 \%$. El $80 \%$ de las atelectasias y los dos casos de neumonía se presentaron en los pacientes con mayor debilidad muscular respiratoria preoperatoria (PImáx. y PEmáx. < $60 \%$ de los valores de referencia). Aquellos pacientes que presentaron una diferencia entre la PImax. posquirúrgica y la PImáx. prequirúrgica superior a $19 \%$ incrementaron el riesgo de atelectasia 10 veces $(O R=10, I C 95 \% 0,85-117,02 ; p=0,067)$. Al alta, el $\mathrm{FEV}_{1}$ fue $29 \%$ menor al valor inicial, la FVC $-32 \%$, la PImáx. -32\%, la PEmáx. -32\%.
Tabla 1 Características clínicas, funcionales y quirúrgicas de los pacientes

\begin{tabular}{|c|c|c|}
\hline Datos Clínicos y Quirúrgicos & $\mathrm{n}$ & $\%$ \\
\hline \multirow{2}{*}{\multicolumn{3}{|c|}{$\begin{array}{l}\text { Clase Funcional Prequirúrgica NYHA (New York Heart } \\
\text { Association) }\end{array}$}} \\
\hline & & \\
\hline 1 & 2 & $7 \%$ \\
\hline 2 & 21 & $70 \%$ \\
\hline 3 & 6 & $20 \%$ \\
\hline 4 & 1 & $3 \%$ \\
\hline \multicolumn{3}{|l|}{ EuroSCORE Logístico (\%) } \\
\hline Mediana & 3,15 & \\
\hline Q1-Q3 & $1,51-6,59$ & \\
\hline \multicolumn{3}{|l|}{ Indice de Charlson } \\
\hline Mediana & 3,4 & \\
\hline Q1-Q3 & $2,5-4,2$ & \\
\hline \multicolumn{3}{|l|}{ Tipo de cirugía realizada } \\
\hline Revascularización miocárdica & 22 & $73 \%$ \\
\hline $\begin{array}{l}\text { Revascularización } \\
\text { miocárdica + cambio de válvula } \\
\text { aórtica }\end{array}$ & 2 & $7 \%$ \\
\hline $\begin{array}{l}\text { Revascularización } \\
\text { miocárdica + Bentall }\end{array}$ & 1 & $3 \%$ \\
\hline Cambio de válvula aórtica & 1 & $3 \%$ \\
\hline $\begin{array}{l}\text { Cambio de válvula aórtica + cambio } \\
\text { de válvula mitral }\end{array}$ & 1 & $3 \%$ \\
\hline Cambio de válvula aórtica + Maze & 1 & $3 \%$ \\
\hline Plastia de válvula mitral & 1 & $3 \%$ \\
\hline $\begin{array}{l}\text { Plastia de válvula } \\
\text { mitral + Maze + cierre Foramen } \\
\text { Oval + Extracción de trombo } \\
\text { auricular }\end{array}$ & 1 & $3 \%$ \\
\hline \multicolumn{3}{|l|}{ Comorbilidades } \\
\hline Hipertensión arterial & 18 & $60 \%$ \\
\hline Dislipidemia & 10 & $33 \%$ \\
\hline Diabetes & 7 & $23 \%$ \\
\hline Obesidad & 3 & $10 \%$ \\
\hline Enfermedad renal crónica & 2 & $6 \%$ \\
\hline Hipotiroidismo & 1 & $3 \%$ \\
\hline Disección aórtica & 1 & $3 \%$ \\
\hline Enfermedad de Chagas & 1 & $3 \%$ \\
\hline Ninguno & 1 & $3 \%$ \\
\hline \multicolumn{3}{|l|}{ Técnica de abordaje quirúrgico } \\
\hline Esternotomía mediana & 29 & $97 \%$ \\
\hline Minitoracotomía & 1 & $3 \%$ \\
\hline \multicolumn{3}{|l|}{ Tiempo de bomba (min) } \\
\hline Mediana & 113 & \\
\hline Q1-Q3 & $97-126$ & \\
\hline
\end{tabular}

\section{Discusión}

Los hallazgos identificados en este estudio sugieren la presencia de alteraciones de la fuerza y resistencia de los músculos inspiratorios y espiratorios en pacientes llevados a la cirugía cardiaca, evidenciados en niveles menores o iguales al $80 \%$ del valor de referencia sugerido por Morales y colaboradores para la presión inspiratoria máxima y la 

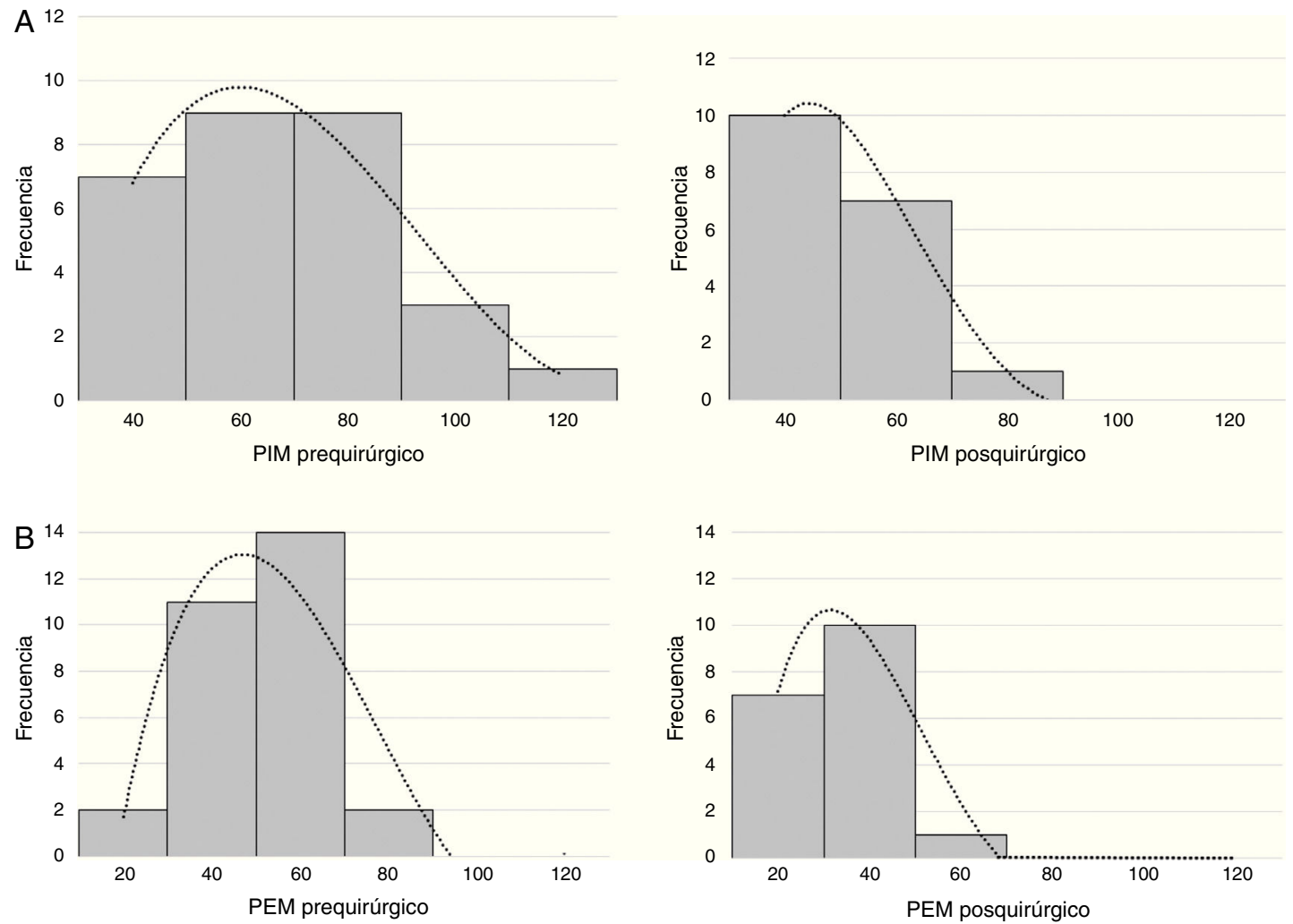

Figura 1 A. Valores basales de la fuerza muscular inspiratoria y sus cambios tras la cirugía cardiovascular. Histogramas de frecuencia para los valores de PImáx. (en $\mathrm{cm} \mathrm{H}_{2} \mathrm{O}$ ) medida antes (prequirúrgico) y después (posquirúrgico). $\mathrm{B}$. Valores basales de la fuerza muscular espiratoria y sus cambios tras la cirugía cardiovascular. Histogramas de frecuencia para los valores de $\mathrm{PEmáx}$. (en $\mathrm{cm} \mathrm{H}_{2} \mathrm{O}$ ) medida antes (prequirúrgico) y después (posquirúrgico).

presión espiratoria máxima ${ }^{21}$. Estos resultados son consistentes con lo observado por otros autores como Morsch y Cols., quienes reportan en su estudio de perfil ventilatorio de los pacientes sometidos a revascularización, una disminución significativa de la fuerza muscular respiratoria en el período postoperatorio, evidenciado en la disminución de la PImáx. y la PEmáx. ${ }^{2}$. De igual manera, Hulzebos y colaboradores, definen como factor protector en su modelo predictivo para el desarrollo de complicaciones pulmonares postoperatorias en la cirugía de revascularización, la presión espiratoria máxima y la capacidad vital inspiratoria con niveles iguales o mayores al $75 \%$ del valor de referencia ${ }^{6,11}$.

La alteración en los valores de las presiones respiratorias supone un factor de riesgo para el desarrollo de las complicaciones pulmonares durante el postoperatorio de la cirugía cardiaca, siendo las atelectasias y las neumonías las más comunes en estos pacientes ${ }^{22}$. Los resultados de este estudio mostraron que el $80 \%$ de las atelectasias y las neumonías fueron presentados en los pacientes con mayor debilidad muscular respiratoria preoperatoria (PImáx. y PEmáx. $<60 \%$ de los valores de referencia) acorde con lo descrito por Bellinetti, quien observó que aquellos pacientes que padecían de una fuerza muscular respiratoria disminuida en el preoperatorio presentaban un riesgo relativo de 5,5 para el desarrollo de las complicaciones pulmonares durante el postoperatorio de la cirugía cardiaca comparando con aquellos pacientes con fuerza muscular conservada ${ }^{12}$. En el presente estudio aquellos pacientes que presentaron una diferencia entre la PImáx. posquirúrgica y la PImáx. prequirúrgica superior a 19\% incrementaron el riesgo de atelectasia 10 veces $(O R=10, I C 95 \% 0,85-117,02 ; p=0,067)$. De igual manera, se ha descrito la relación entre la presión inspiratoria máxima y la duración de la estancia hospitalaria. Guedes y Cols., refieren una correlación negativa entre las dos y sugieren que la presión inspiratoria máxima mayor o igual al $75 \%$ del valor de referencia puede ser considerada como un factor protector en la reducción de la estancia durante el postoperatorio de la cirugía torácica ${ }^{8}$.

Otro elemento importante a evaluar en este estudio fue la capacidad espirométrica de los pacientes, la cual estuvo disminuida en un $54 \%$ de los casos (35\% debido a alteración obstructiva, $19 \%$ no-obstructiva; $\mathrm{FEV}_{1}$ entre $32-76 \%$ ref.). Esta disminución empeoró con el transcurrir de la estancia hospitalaria: al alta, el volumen espiratorio forzado en el primer segundo (FEV1) fue $29 \%$ menor y la capacidad vital forzada (FVC) -32\%, al igual que lo referido por Morsch y colaboradores, quienes encontraron una reducción significativa en estos niveles de volumen espiratorio, medidas desde el preoperatorio hasta el día 6 del postoperatorio, en pacientes llevados a la cirugía de revascularización miocárdica electiva ${ }^{2}$.

La detección de las alteraciones respiratorias en la fuerza y resistencia de los músculos respiratorios, así como en la función pulmonar en el preoperatorio de la cirugía cardiovascular, podrían retardar las intervenciones quirúrgicas cardiovasculares. No obstante, una detección temprana y 
oportuna de estas alteraciones en el preoperatorio conducirían a la disminución de la incidencia y prevalencia de las complicaciones pulmonares en el postoperatorio de la cirugía cardiaca contribuyendo en la disminución de la morbimortalidad, la estancia hospitalaria y los costos o recursos utilizados en estos pacientes. En este sentido, los estudios han demostrado la eficacia del entrenamiento preoperatorio de los músculos respiratorios para prevenir la aparición de complicaciones pulmonares en esta población ${ }^{23}$. Hulzebos y Cols., demostraron a través del entrenamiento de los músculos inspiratorios haciendo uso de la válvula Threshold ${ }^{\circledR}$ en el preoperatorio de revascularización (realizado dos semanas antes de ser llevado a cabo el procedimiento quirúrgico), una disminución significativa de la incidencia de estas complicaciones y de la estancia hospitalaria durante el postoperatorio en pacientes con alto riesgo de desarrollarlas ${ }^{11}$. Así mismo, Dall'Ago y Cols., evidenciaron un incremento del $115 \%$ de la PIM, del $17 \%$ de la captación de oxígeno y del $19 \%$ de la distancia recorrida en el test de 6 minutos, en los pacientes con falla cardiaca y debilidad de los músculos inspiratorios, sometidos a un entrenamiento de sus músculos inspiratorios durante 12 semanas $^{24}$, lo que sugiere, además, de una identificación de las alteraciones, la posibilidad de una intervención precoz.

Con base a los hallazgos identificados en este estudio y a los resultados documentados en la literatura, se propone no solo establecer un protocolo para la detección oportuna de dichas alteraciones, sino también explorar las posibles intervenciones que sean factibles para su realización en el preoperatorio de la cirugía cardiaca. Igualmente, las intervenciones realizadas durante el cuidado del paciente en el postoperatorio, como la traqueostomía temprana, podría ser considerada en aquellos pacientes que sumado a la identificación de la disfunción muscular respiratoria en el prequirúrgico, requieran de ventilación mecánica prolongada. Finalmente, el uso de fármacos anabólicos y sus beneficios en pacientes con atrofia muscular ha sido estudiado recientemente, obteniéndose diferencias significativas comparadas con quienes no reciben dicha intervención, abriendo nuevos campos en la investigación de este tema ${ }^{24}$. Se recomienda en futuras investigaciones explorar los cambios subyacentes a la disfunción muscular, a través de la toma de biopsias musculares en estos pacientes, así como la exploración de futuras intervenciones de entrenamiento muscular respiratorio.

Este estudio tuvo una limitante dada por la disponibilidad de un solo equipo para la medición de las presiones inspiratoria y espiratoria, y la espirometría, por lo cual se dificultó la realización de algunas mediciones planeadas e impidió una mayor recolección de mediciones en estos pacientes.

\section{Conclusiones}

Los pacientes llevados a la toracotomía por enfermedad cardiaca tienen una insospechada disfunción de los músculos respiratorios que empeora con la cirugía y la hospitalización, y que se asocia a un incremento del riesgo de complicaciones respiratorias. El entrenamiento muscular y la administración de fármacos anabolizantes podrían tener efectos beneficiosos en los pacientes de mayor riesgo de complicaciones postoperatorias.

\section{Responsabilidades éticas}

Protección de personas y animales. Los autores declaran que los procedimientos seguidos se conformaron a las normas éticas del comité de experimentación humana responsable y de acuerdo con la Asociación Médica Mundial y la Declaración de Helsinki.

Confidencialidad de los datos. Los autores declaran que han seguido los protocolos de su centro de trabajo sobre la publicación de datos de pacientes.

Derecho a la privacidad y consentimiento informado. Los autores han obtenido el consentimiento informado de los pacientes y/o sujetos referidos en el artículo. Este documento obra en poder del autor de correspondencia.

\section{Financiación}

Estudio INNOTORIO, COLCIENCIAS contrato 744-2013, código 656656933786; y Plan de Fortalecimiento COLCIENCIAS contrato 734-2013.

\section{Conflicto de intereses}

Los autores declaran no tener ningún conflicto de intereses.

\section{Anexo. Material adicional}

Se puede consultar material adicional a este artículo en su versión electrónica disponible en doi:10.1016/ j.rccar.2016.05.014.

\section{Bibliografía}

1. Barreto T, Aragão V, Serra F, Ribeiro D. Influence of respiratory muscle strength in evolution of patients with heart failure after cardiac surgery. Rev Bras Cir Cardiovasc. 2011;26:355-63.

2. Morsch K, Leguisamo C, Camargo M, Coronel C, Mattos W, Ortiz $\mathrm{L}$, et al. Ventilatory profile of patients undergoing CABG surgery. Rev Bras Cir Cardiovasc. 2009;24:180-7.

3. Verissimo P, Timenetsky K, Casalaspo J, Rodrigues L, Yang A, Caserta R. High Prevalence of Respiratory Muscle Weakness in Hospitalized Acute Heart Failure Elderly Patients. PLoS One. 2015;10.

4. Martínez-Vázquez J, Torres L, Prevalencia del dolor postoperatorio. Alteraciones fisiopatológicas y sus repercusiones. Rev. Soc. Esp. Dolor. 2000;7:465-76.

5. Barros G, Santos C, Granado B, Costa P, Límaco R, Gardenghi G. Respiratory muscle training in patients submitted to coronary arterial bypass graft. Rev Bras Cir Cardiovasc. 2010;25:483-90.

6. Hulzebos EH, Helders PJ, Favié NJ, De Bie RA, Brutel de la Riviere A, Van Meeteren NL. Preoperative Intensive Inspiratory Muscle Training to Prevent Postoperative Pulmonary Complications in High-Risk Patients Undergoing CABG SurgeryA Randomized Clinical Trial. JAMA. 2006:296. No. 15.

7. Oikkonen M, Karjalainen K, Kahara V, Ktiosa R, Schavikin L. Comparison of Incentive Spirometry and Intermittent Positive Pressure Breathing after Coronary Artery Bypass Graft. Chest. 1991;99:60-5.

8. Guedes GP. Correlação entre força muscular respiratória e otempo de internação pós-operatório. Fisioter Mov. 2009;22:605-14. 
9. Bowen T, Rolim N, Fischer T, Bakkerud F, Medeiros A, Werner $S$, et al. Heart failure with preserved ejection fraction induces molecular, mitochondrial, histological, and functional alterations in rat respiratory and limb skeletal muscle. Eur J Heart Fail. 2015;17:263-72.

10. Cahalin L, Sernigran M, Dec GW. Inspiratory Muscle Training in Patients With Chronic Heart Failure Awaiting Cardiac Transplantation: Results of a Pilot Clinical Trial. Phys Ther. 1997;77:830-8.

11. Hulzebos E, Van-Meeteren N, De Bie R, Dagnelie P, Helders P. Prediction of Postoperative Pulmonary Complications on the Basis of Preoperative Risk Factors in Patients Who Had Undergone Coronary Artery Bypass Graft Surger. Phys Ther. 2003;83:8-16.

12. Bellinetti LM, Thomson JC. Avaliação muscular respiratória nas toracotomias e laparotomias superiores eletivas. J Bras Pneumol. 2006;32:99-105.

13. Rodrigues A, Mendes V, Gomes P, Arruda M, Xavier F, Santos V, et al. Preoperative respiratory muscle dysfunction is a predictor of prolonged invasive mechanical ventilation in cardiorespiratory complications after heart valve surgery. Eur J Cardiothorac Surg. 2011;39:662-6.

14. Gomes P, Rodrigues A, Barboza P. Effects of an Inspiratory Muscle Rehabilitation Program in the Postoperative Period of Cardiac Surgery. Arq Bras Cardiol. 2009;92:275-82.

15. Nomori H, Kobayashi R, Fuyun G, Morinaga S, Yashima H. Assessment in Thoracic Surgery Patients With Special Reference to Postoperative Pulmonary Complications. Chest. 1994;105:1782-8.
16. Muñoz-Blanco F, Salmerón J, Santiago J, Marcote C. Complicaciones del dolor postoperatorio Rev. Soc. Esp. Dolor. 2001;8:194-211.

17. Manual SEPAR de Procedimientos. Procedimientos de evaluacion de la función pulmonar. Disponible en: https: / / issuu.com/ separ/docs/procedimientos4/125

18. The Society of Thoracy Surgeons. Adult Cardiac Surgery Database, v2.61 Training Manual http://www.sts.org/sites/ default/files/documents/pdf/trainingmanuals/adult2.61/ Section_P_COMPLICATIONS.pdf

19. Evans JA, Whitelaw WA. The assessment of maximal respiratory mouth pressures in adults. Respir Care. 2009;54:1348-59.

20. Hautmann H, Hefele S, Schotten K, Huber RM. Maximal inspiratory mouth pressures (PIMAX) in healthy subject what is the lower limit of normal? Respir Med. 2000;94:689-93.

21. Morales P, Sanchis J, Cordero P-J, Dies JL, Presiones respiratorias estáticas en adultos. Valores de referencia para población caucásica mediterránea. Arch Bronconeumol. 1997;33:213-9.

22. Meyer F, Borst M, Zugck C, Kirschke A, Schellberg D, Kübler W, et al. Respiratory Muscle Dysfunction in Congestive Heart Failure Clinical Correlation and Prognostic Significance Circulation. 2001;103:2153-8.

23. Vasques R, Trevizan S, Rodrigues C. Respiratory physiotherapy and its application in preoperative period of cardiac surgery. Rev Bras Cir Cardiovasc. 2011;26:647-52.

24. Dall'Ago P, Chiappa GR, Guths H, Stein R, Ribeiro JP. Inspiratory muscle training in patients with heart failure and inspiratory muscle weakness: a randomized trial. J Am Coll Cardiol. 2006;47:757-63. Epub 2006 Jan 26. 